NASA Technical Memorandum 105694

AIAA-92-3090

\title{
Experimental Study of Cross-Stream Mixing in a Rectangular Duct
}

D.S. Liscinsky and B. True

United Technologies Research Center

East Hartford, Connecticut
A. Vranos
A.B. Research Associates
South Windsor, Connecticut

and

J.D. Holdeman

Lewis Research Center

Cleveland, Ohio

Prepared for the

28th Joint Propulsion Conference and Exhibit

cosponsored by the AIAA, SAE, ASME, and ASEE

Nashville, Tennessee, July 6-8, 1992 


\title{
EXPERIMENTAL STUDY OF CROSS-STREAM MIXING IN A RECTANGULAR DUCT
}

\author{
D.S. Liscinsky ${ }^{*}$ and B. True ${ }^{* *}$ \\ United Technologies Research Center \\ East Hartford, CT 06108 \\ A. Vranos ${ }^{\dagger}$ \\ AB Research Associates \\ South Windsor, CT 06074 \\ J.D. Holdeman ${ }^{\dagger \dagger}$ \\ National Aeronautics and Space Administration \\ Lewis Research Center \\ Cleveland, $\mathrm{OH} \quad 44135$
}

\section{$\underline{\text { Abstract }}$}

An experimental investigation of non-reacting cross-stream jet injection and mixing in a rectangular duct has been conducted with application to a low emissions combustor. Planar digital imaging was used to measure concentration distributions in planes perpendicular to the duct axis. Mixing rate was measured for $45^{\circ}$ slanted slot and round orifice injectors. It is shown that (1) mixing improves continuously with increasing momentumflux ratio, (2) given a momentum-flux ratio, there is an optimum orifice spacing, (3) mixing is more dependent on injector geometry than mass flow ratio, (4) mixing is influenced by relative slot orientation, and (5) jet structure is different for round holes and slanted slots injectors. The utility of acquiring multipoint fluctuating properties of the flow field is also demonstrated.

\section{Nomenclature}

$\mathrm{C}_{\text {avg }}$ fully mixed mass fraction $=\left(w_{j} / w_{m}\right) /\left(1+w_{j} / w_{m}\right)$ $=\theta_{\mathrm{EB}}$, (Ref. 1)

$\mathrm{H}$ duct height at injection plane $=4$ in

$\mathrm{J}$ jet-to-mainstream momentum-flux ratio $=\left(\rho_{j} V_{j}^{2}\right) /\left(\rho_{m} V_{m}^{2}\right)$

$\mathrm{L}$ length of the orifice, longest dimension (see Table 1)

$\mathrm{S} \quad$ spacing between orifice mid-points

$\mathrm{U}_{\mathrm{S}} \quad$ spatial unmixedness parameter (Eq. 1)

$\mathrm{V}_{\mathrm{m}} \quad$ mainstream velocity $=10 \mathrm{ft} / \mathrm{s}$

$\begin{array}{ll}* & \text { Research Scientist, Member AIAA } \\ * * & \text { Senior Laboratory Technician } \\ \dagger & \text { Consultant, Member AIAA } \\ \dagger \dagger & \text { Senior Research Engineer, Senior Member AIAA }\end{array}$

\section{Nomenclature (cont.)}

$\mathrm{V}_{\mathrm{j}} \quad$ jet velocity $=\mathrm{m}_{\mathrm{j}} / \rho_{\mathrm{j}} \mathrm{A}_{\mathrm{j}} \mathrm{C}_{\mathrm{d}}$

$\mathrm{W}$ width of the orifice, shortest dimension (see Table 1)

$w_{j} / w_{m}$ jet-to-mainstream mass flow ratio

$\mathrm{X}$ downstream location, $\mathrm{X}=0$ at the upstream edge of the orifice

\section{Introduction}

Cross-flow mixing is common to many practical commercial processes. In particular, the study of jets injected perpendicular to a confined cross flow has received a great deal of attention for application to the dilution zone of gas turbine combustors. $^{2}$ Interest continues in the cross-flow mixing problem with the implementation of stringent air quality regulations which require development of combustors with lower emissions. A critical technology in one of these concepts, a Rich-Burn/Quick-Mix/Lean-Burn(RQL) combustor, is the rapid and uniform mixing to control emissions. ${ }^{4}$

Regardless of the motivating application, study of the turbulent mixing process requires characterization of timeresolved fluid concentration as a function of spatial position. Laser-based optical diagnostics use light scattering for in situ measurement of concentration. Planar light scattering techniques consisting of simultaneous, multipoint measurements allow visualization of the flow field, study of large-scale turbulent structure and determination of statistical properties. ${ }^{3}$ Concentration fluctuations and spatial characteristics of the flow obtained using planar diagnostics can be used to relate mixing uniformity to potential emissions. 


\section{Experimental}

\section{Apparatus}

Figure 1 is a schematic representation of the apparatus. The apparatus consists of 3 parallel contiguous ducts of rectangular cross section, simulating a sector of an annular combustor. Sector width is 12 inches. The inner duct height is 4 inches. The outer ducts (shrouds), which supply the injectant gas, are 1 inch in height. These are separated from the inner duct by removable 0.12 inch thick flat plates. The injectant is fed from the shrouds to the inner duct through orifices of various sizes and shapes that are machined into the plates. Mass flow to each of the 3 ducts is controlled independently using venturi flowmeters. The maximum variation in the mean approach velocity of the mainstream flow was $6 \%$ with a turbulence level of $1.3 \%$.

\section{Data Acquisition}

Planar digital imaging was used to measure optically concentration distributions in planes perpendicular to the duct axis $1,2,3$, and 4 inches downstream of the midpoint of the orifice for each test configuration and flow condition. The laser light scattering technique, based on Mie-scattering, can be summarized as follows: The jet flow is marked with an oil aerosol ( $\mu \mathrm{m}$ sized particles). A light sheet ( 0.02 inch thick) is created using a $2 \mathrm{~W}$ argon-ion laser and a rotating mirror. The flow field is illuminated by passing the light sheet through a window in the sidewall of the test section. A solid state camera, located inside the duct $2.5 \mathrm{ft}$ downstream of the orifice centerline, is focused on the illuminated plane (end-on view). The camera is programmed to make exposures coincident with the sweep of the beam through the flow field. The image is digitized and sent to a computer for storage. The scattered light intensity is proportional to the number of particles in the measurement volume. If only one of two streams is marked, the light intensity of the undiluted marked fluid represents mole fraction unity. For a more detailed discussion of the technique see Ref. 7.

An image intensified, thermo-electrically cooled, CCD camera was used to record the images. Time-averaged ( 5 second exposure) measurements of the concentration distribution were obtained for all tests. Time-resolved $(50 \mu \mathrm{s}$ exposure) measurements were obtained for two configurations: opposed rows of inline slanted slots (code SSE in Table 1) and opposed rows of staggered slanted slots (code SSG in Table 1). All images recorded the entire duct cross section at a spatial resolution of $0.02 \times 0.02 \times 0.02$ inches in a data frame containing 110,592 pixels (576 x 192 format).

\section{Data Reduction/Analysis}

In order to compute absolute mole fraction distribution from the digital image, the mole fraction at at least one point in the flow field must be known. This can be done using a hydrocarbon tracer and a sampling probe ${ }^{8}$, but for these experiments a simple approximation was made. The fully mixed mole fraction, $\mathrm{C}_{\mathrm{avg}}$, is determined from the metered mass flows. Since the scattered light intensity distribution across the entire duct is known for each frame, the average light intensity in each image is proportional to $\mathrm{C}_{\mathrm{avg}}$. Each pixel in the image was subsequently normalized to obtain a local average mole fraction. It is shown in Ref. 8 that this is an acceptable approximation.

\section{Mean concentration distributions, i.e. spatial} distributions of the local mean concentration, can be used to define the mixing effectiveness of each configuration. In a previous paper $^{8}$, an unmixedness parameter was defined

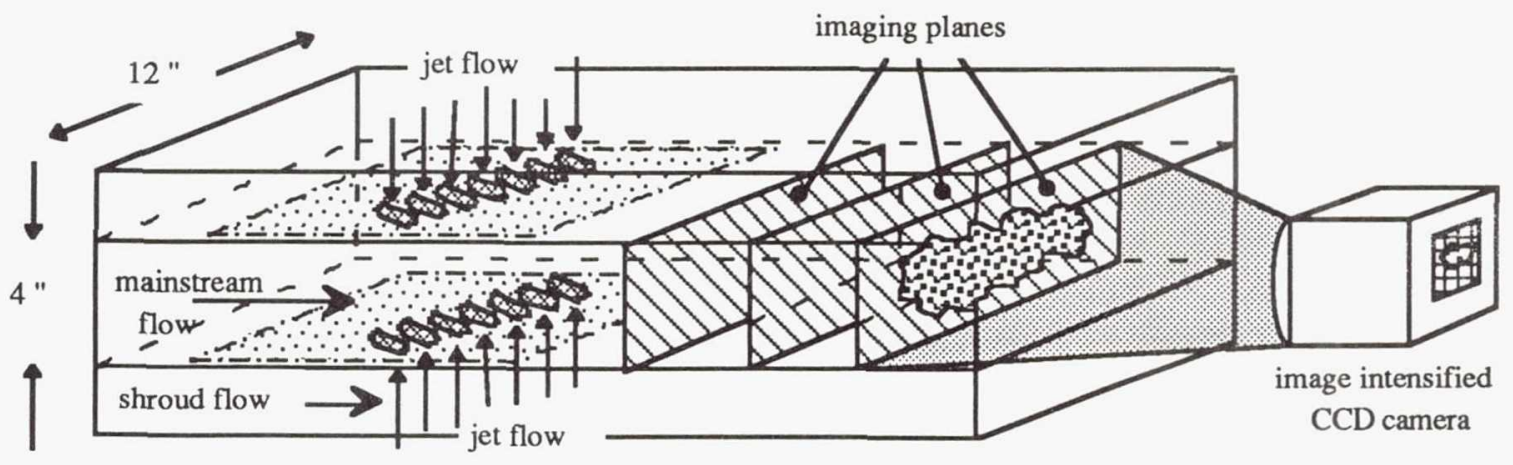

Figure 1: Experimental Configuration used to Measure Planar Concentration Distributions 
to allow rapid screening of a variety of flow and geometric configurations in a cylindrical duct. The same spatial unmixedness parameter, $\mathrm{U}_{\mathrm{S}}$, defined by Eq. 1, was used in this investigation. In a perfectly mixed system $\mathrm{U}_{\mathrm{S}}=0$.

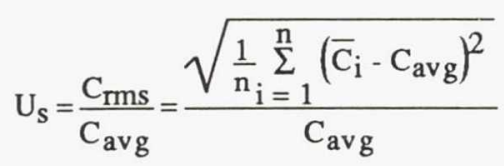

where,

$$
\begin{aligned}
& \mathrm{n}=\text { number of pixels in each image } \\
& \overline{\mathrm{C}}_{\mathrm{i}}=\text { average concentration at a pixel } \\
& \mathrm{C}_{\mathrm{avg}}=\frac{1}{\mathrm{n}} \sum_{\mathrm{i}=1}^{\mathrm{n}} \overline{\mathrm{C}}_{\mathrm{i}}
\end{aligned}
$$

\section{Mixing Configurations}

Table 1 identifies the 7 orifice plate configurations tested. The configuration sketches are drawn approximately $1 / 2$ scale in Table 1. Two injector shapes were studied: (1) slanted slots, aspect ratio $4: 1$, and (2) round holes. The slots had circular ends and were slanted $45^{\circ}$ to the mainstream flow direction. Injection was 2 -sided. Discharge coefficients were measured for each orifice shape and used to set 4 momentum-flux ratios $(\mathrm{J})$ : $16,36,64,100$. The mainstream flow was held constant at $10 \mathrm{ft} / \mathrm{sec}$ for all tests.

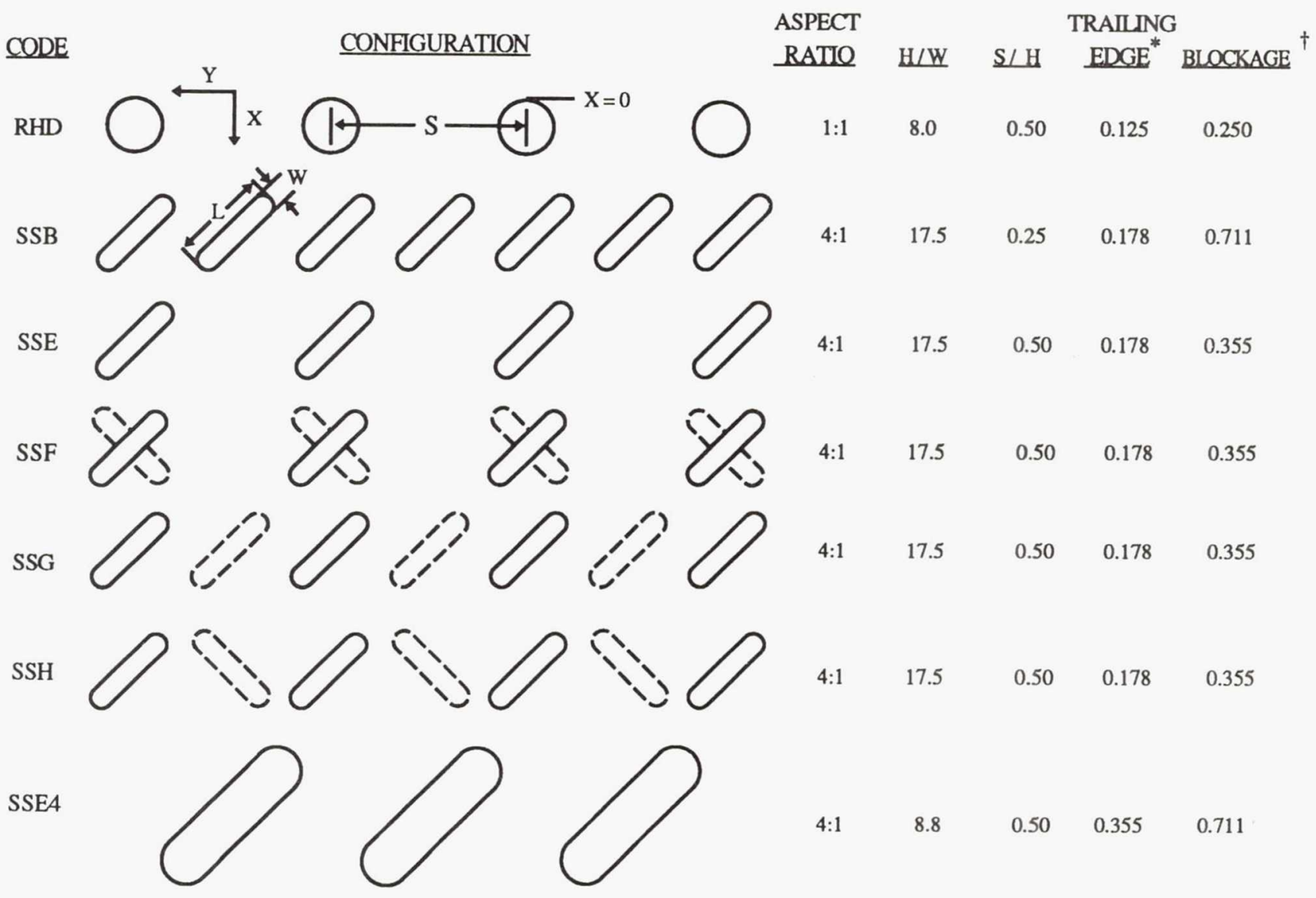

Table 1: Orifice Plate Configurations

(dotted lines represent orifices on the opposite wall)

${ }^{*} \mathrm{X}$ projection $/ \mathrm{H}(\mathrm{H}=4$ inches for all tests $)$

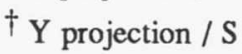




\section{Results and Discussion}

\section{Average Concentration Distributions}

Distributions of average mole fraction are shown as a function of downstream distance at momentum-flux ratios of 16 and 36 in Fig. 2. The orifice configuration consists of opposed rows of inline slanted slots (code SSE in Table 1). The top and bottom slots slant in the same direction (parallel). The individual jets are still discernible at the first downstream location. In contrast to a round orifice jet (Fig. 7), the slanted slot jet forms a pair of counter-rotating vortices which are of unequal size and strength. The vortex formed at the upstream edge of the slot is larger and penetrates farther than the vortex formed at the downstream edge. The vortex pair is rotated so that the bulk of jet fluid is found toward the side of the slot that is upstream, thereby identifying the direction in which the slot is slanted (In Fig. 2 the upstream edge of the slot is on the right). In addition, the main body of the jet is displaced relative to the midpoint of the slot.

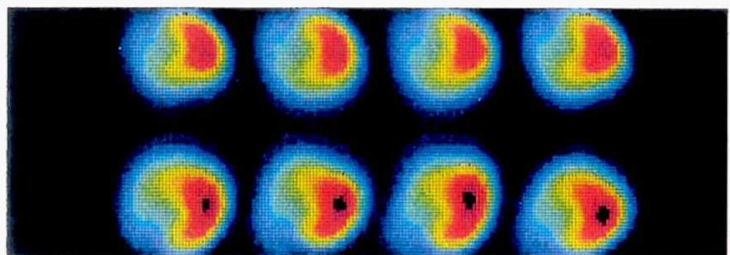

mole

fraction

0.50
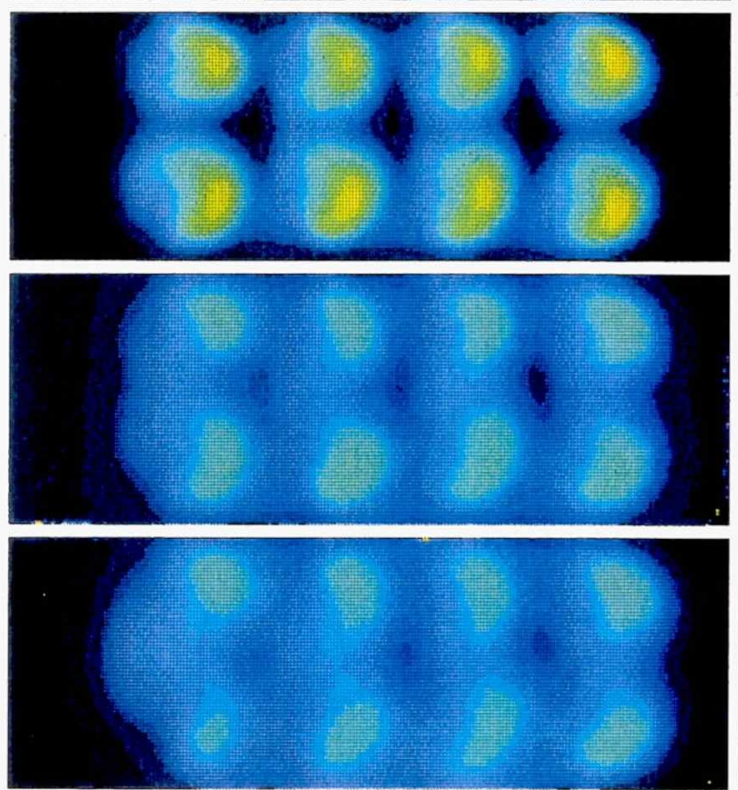

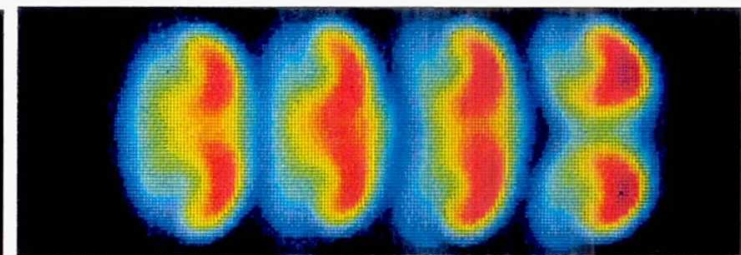

$\mathrm{X} / \mathrm{H}=0.33$

\section{Effect of Flow Conditions}

The effect of momentum-flux ratio on unmixedness is shown in Fig. 3 for a row of opposed inline slanted slots (code SSE in Table 1). Mixing rate was found to increase continuously with increasing $J$. None of the test configurations showed an optimum value of $\mathrm{J}$ over the range studied.

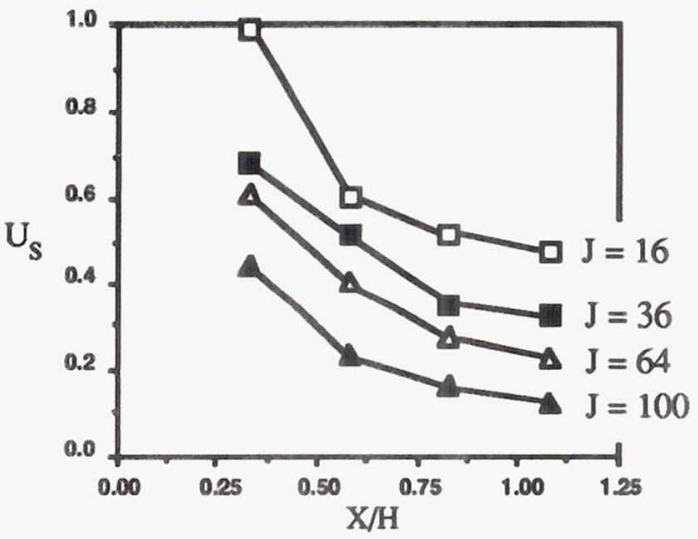

Figure 3: Effect of Momentum-flux ratio on Opposed Inline Slanted Slots, $\mathrm{S} / \mathrm{H}=0.5$, code SSE in Table 1
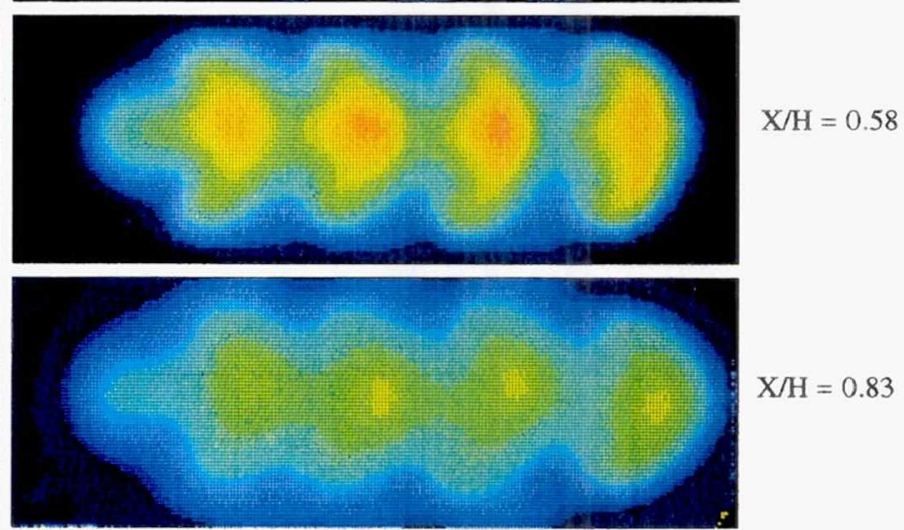

$\mathrm{X} / \mathrm{H}=0.83$

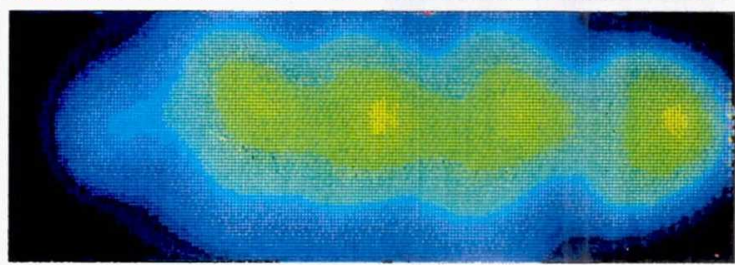

$\mathrm{X} / \mathrm{H}=1.08$

Figure 2: Average Concentration Distributions of the Flow through Opposed Inline $45^{\circ}$ Slanted Slots, code SSE in Table 1 
A value of $\mathrm{U}_{\mathrm{S}}$ below 0.1 was not observed in any configuration. In most configurations there was a noticeable decrease in mixing rate beyond $\mathrm{X} / \mathrm{H}=0.5$. In some cases the change in $\mathrm{U}_{\mathrm{S}}$ from $\mathrm{X} / \mathrm{H}=0.8$ to $\mathrm{X} / \mathrm{H}=1.0$ was not significant. Therefore, the initial mixing rate is vital in emissions control.

\section{Effect of Geometry}

\section{(1) Orifice Spacing}

$\mathrm{U}_{\mathrm{S}}$ is shown as a function of orifice spacing at $\mathrm{J}=36$ for opposed rows of inline slanted slots in Fig 4. Mixing improved at closer spacing. Previously, Holdeman ${ }^{2}$, has shown, on the basis of mean temperature distributions, that for multi-source injection from equally spaced round orifices in rows, mixing can be correlated by a single variable composed of the product of momentum-flux ratio and orifice spacing, independent of orifice size. Optimum penetration and mixing, for any given configuration, is obtained when momentum-flux ratio and orifice spacing are inversely proportional such that:

$$
\sqrt{ } \mathrm{J} * \mathrm{~S} / \mathrm{H}=\mathrm{C}
$$

where,

$$
\begin{aligned}
& \mathrm{S}=\text { spacing between orifice mid-points } \\
& \mathrm{H}=\text { duct height } \\
& \begin{aligned}
\mathrm{J} & =\text { momentum-flux ratio } \\
\mathrm{C} & =2.50 \text { for single side injection } \\
& =1.25 \text { for } 2 \text {-sided opposed inline injection } \\
& =5.00 \text { for } 2 \text {-sided opposed staggered injection }
\end{aligned}
\end{aligned}
$$

From Eq. 2, at $\mathrm{J}=36$, the optimum $\mathrm{S} / \mathrm{H}=0.21$. The trend of the data in Fig. 5 supports the value calculated using Eq. 2, in that mixing was better at $\mathrm{S} / \mathrm{H}=0.25$. The inline slots at $\mathrm{S} / \mathrm{H}=0.5$ are more widely spaced than the optimum, and mixing is slower as shown in Fig. 4 and confirmed by the distributions shown for $\mathrm{J}=36$ in Fig. 2. Holdeman ${ }^{2}$ has attributed this phenomenon to over-penetration at wide spacing.

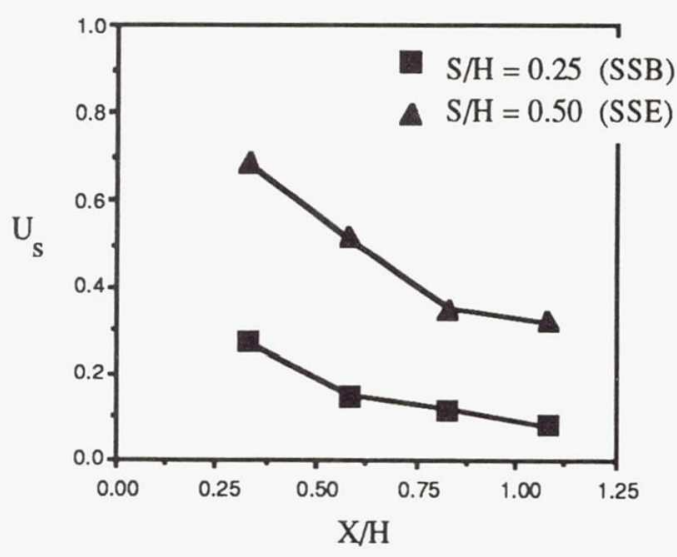

Figure 4: Effect of Orifice Spacing on Unmixedness for Opposed Inline Slanted Slots, J = 36

(codes are from Table 1)

\section{(2) Orifice Size}

The effect of orifice size is shown in Fig 5 for opposed inline slanted slots (code SSE and SSE4 in Table 1). The slot area was enlarged by a factor of 4 while maintaining the same aspect ratio $(4: 1)$, orifice spacing $(\mathrm{S} / \mathrm{H})$ and momentum-flux ratio. Mixing improved as orifice size increased. However, when the smaller orifices (code SSB in Table 1) were tested with the same blockage (defined in Table 1) and J, i.e. a geometrically similar injector configuration, $\mathrm{U}_{\mathrm{S}}$ is the same. Therefore, it may be that the improvement evident in Fig. 5 is due to a smaller blockage between adjacent slots rather than a change in orifice size. In other words, it appears that injector geometry and distribution are controlling the mixing rate rather than mass flow ratio. The results indicate that blockage is an important factor for non-symmetrical orifices, in addition to the parameter $\mathrm{S} / \mathrm{H}$.

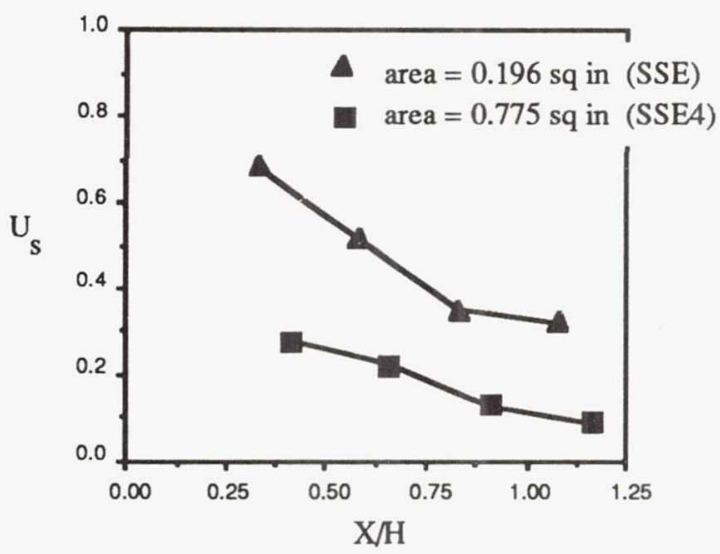

Figure 5: Effect of Orifice Size on Unmixedness for Opposed Inline Slanted Slots, $\mathrm{J}=36$ (codes are from Table 1) 
(3) Orifice Shape

The effect of orifice shape is shown in Fig. 6 . Unmixedness is shown for inline slanted slots (code SSE in Table 1) and round holes (code RHD in Table 1) at $\mathrm{J}=16,36$, and 100. The orifice spacing $(\mathrm{S} / \mathrm{H})$ and mass flow ratios are equal, however the blockage of the round holes is $20 \%$ less. $\mathrm{U}_{\mathrm{S}}$ was found to be about the same for both configurations. Given the different initial vortex structure of the jet fluid (illustrated in Figs. 2 and 7) different mixing rates might have been expected for the two orifice shapes. However, vortex development may only effect mixing when the orifices are more closely spaced so that interaction of the adjacent jets is stronger. This would be the case at the optimum spacing, $\mathrm{S} / \mathrm{H}=0.21$ for $\mathrm{J}=36$.

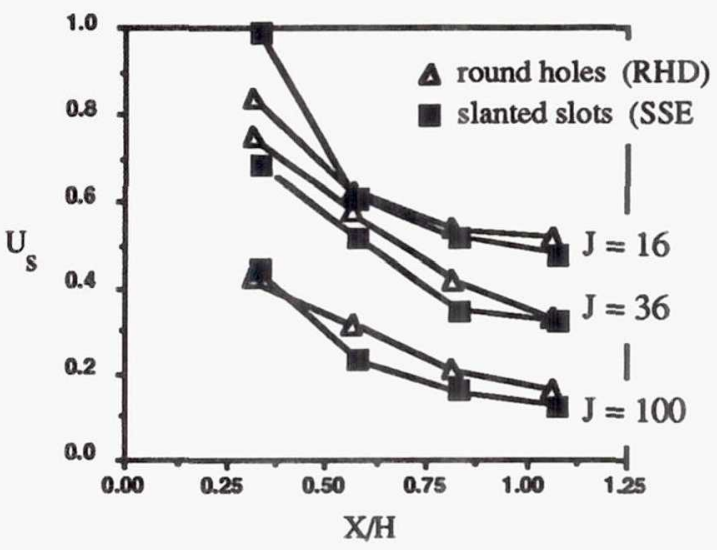

Figure 6: Effect of Orifice Shape on Unmixedness for Opposed Inline Orifices, $\mathrm{S} / \mathrm{H}=\mathbf{0 . 5}$ (codes are from Table 1)
$\mathrm{J}=16$
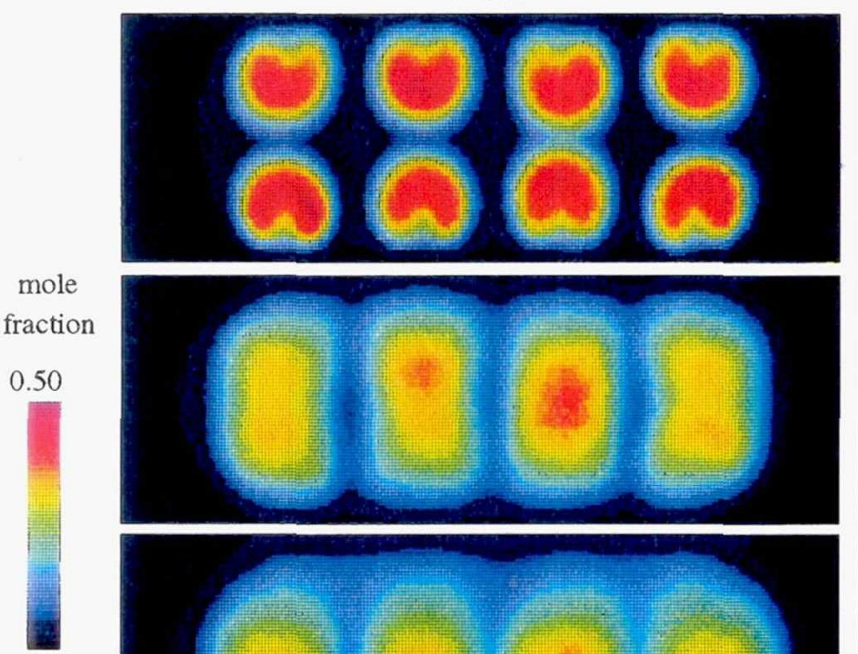

0.0

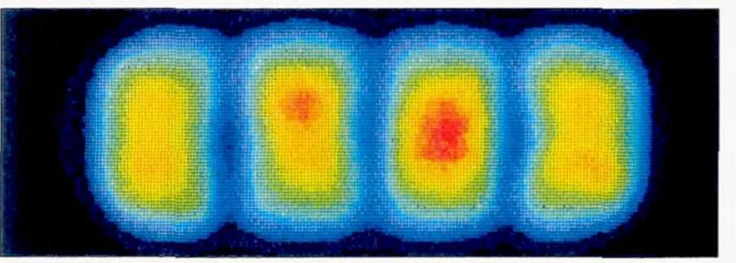

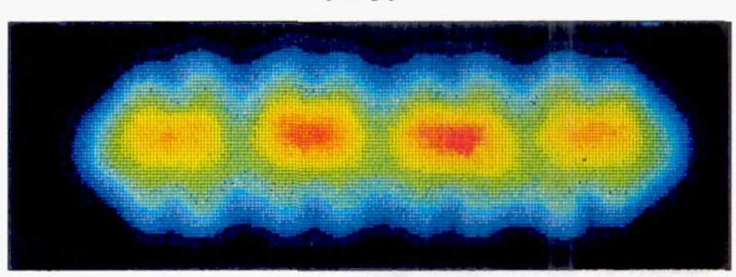

$\mathrm{X} / \mathrm{H}=0.31$

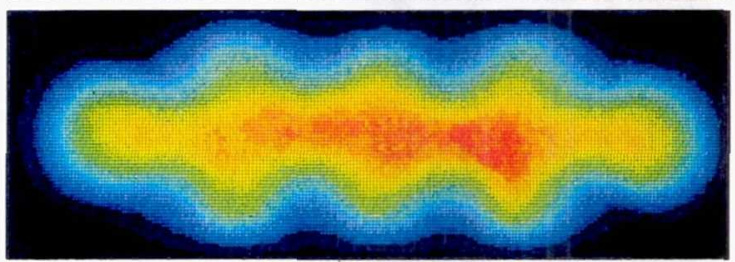

$\mathrm{X} / \mathrm{H}=0.56$
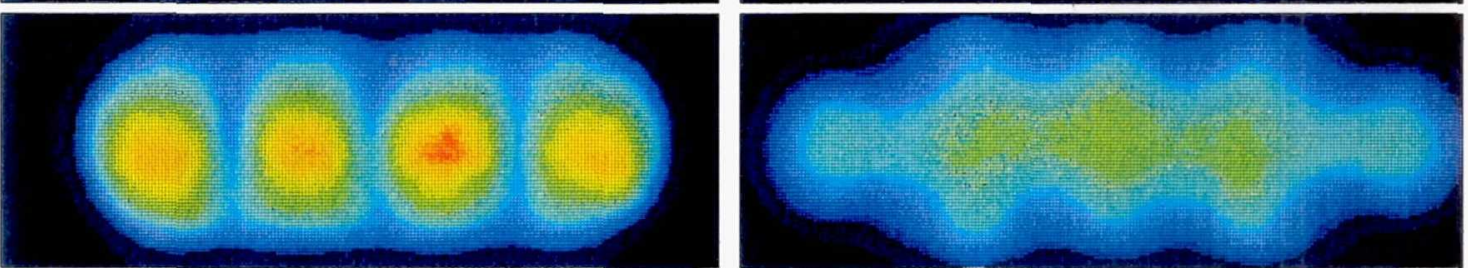

$\mathrm{X} / \mathrm{H}=0.81$
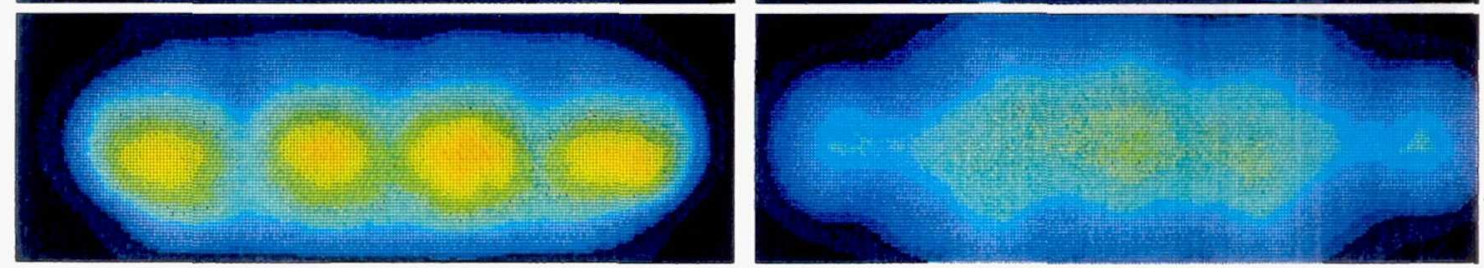

$\mathrm{X} / \mathrm{H}=1.06$

Figure 7: Concentration Distributions of the Flow through

Opposed Inline Round Holes, code RHD in Table 1 
(4) Slot Orientation

Mixing rate can be modified by slot orientation, that is by slanting the slots on the top and bottom duct walls in opposite directions relative to each other ("crossed"). The effect of the "crossed" configuration is shown in Fig. 8 for $\mathrm{J}=36$ and $\mathrm{S} / \mathrm{H}=0.5$. When the slots are inline and "crossed" as in code SSF in Table $1, \mathrm{U}_{\mathrm{S}}$ was the same as if the slots were inline and "parallel" as in code SSE in Table 1. However, when the slots are staggered and "crossed" as in code SSH in Table 1, $U_{S}$ was significantly higher than if the staggered slots were "parallel" as in code SSG in Table 1.

As discussed previously, and as shown in Fig. 9a (code SSE), the main body of the jet from a slanted slot is displaced relative to the midpoint of the orifice. Therefore, in a staggered configuration "crossing" the slots will tend to align, rather than stagger, the jets as shown by codes SSG and SSH in Fig. 9a. As predicted by Eq. 2, an $\mathrm{S} / \mathrm{H}=0.5$ is not optimum for an inline configuration, but closer to the optimum $\mathrm{S} / \mathrm{H}=0.8$ for a staggered configuration. By "crossing" the slots the advantage of the staggered configuration at this $\mathrm{S} / \mathrm{H}$ was lost, as shown by comparing the data from codes SSG and SSH in Fig. 9b.

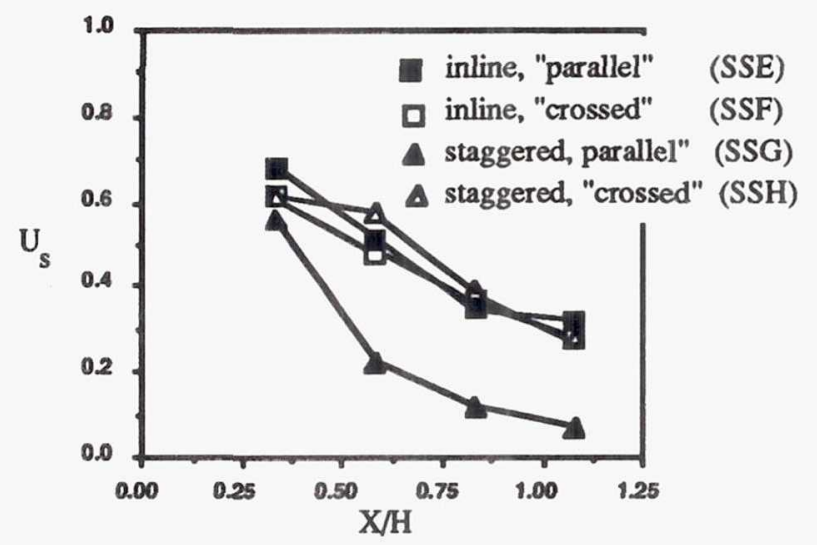

Figure 8: Effect of Slot Orientation on Unmixedness for opposed slanted slots, $\mathrm{J}=36, \mathrm{~S} / \mathrm{H}=0.5$ (codes are from Table 1)

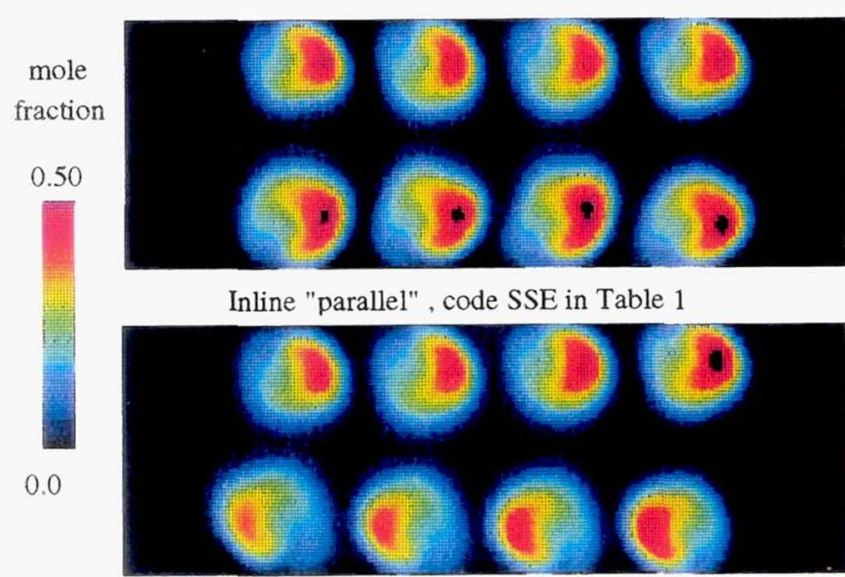

Inline "crossed" , code SSF in Table 1

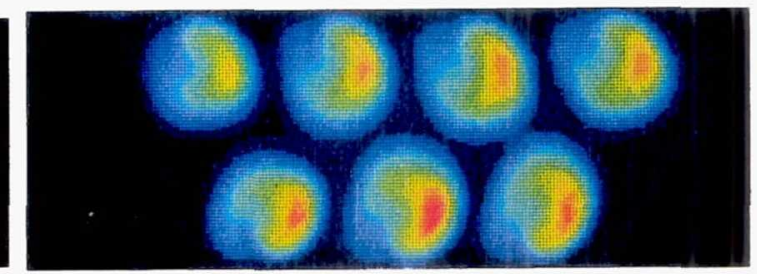

Staggered "parallel", code SSG in Table 1

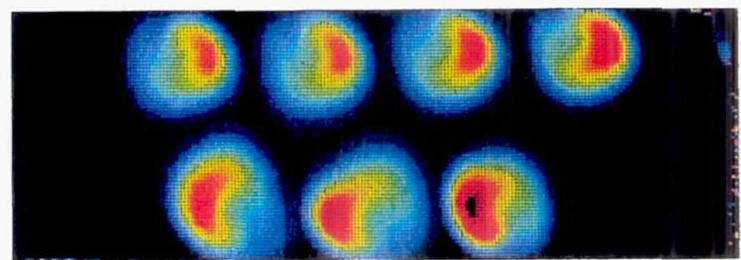

Staggered "crossed" , code SSH in Table 1

Figure 9a: Concentration Distributions of Opposed Slanted Slots at $\mathrm{J}=16, \mathrm{~S} / \mathrm{H}=0.5, \mathrm{X} / \mathrm{H}=0.33$

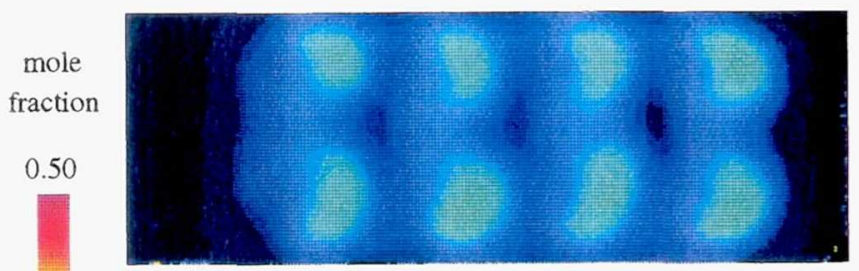

Inline "parallel", code SSE in Table 1

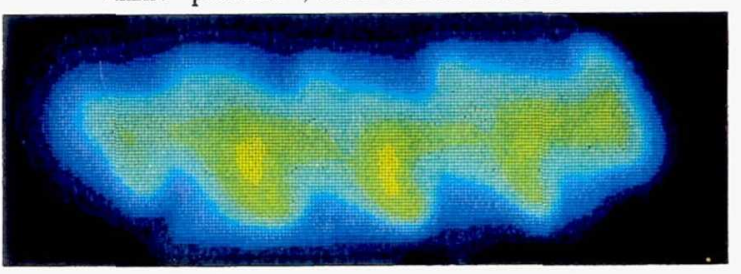

Inline "crossed" , code SSF in Table 1

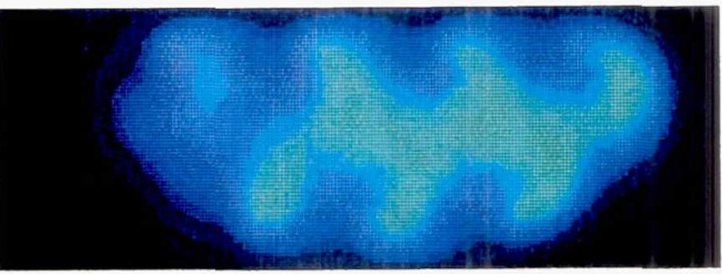

Staggered "parallel" , code SSG in Table 1

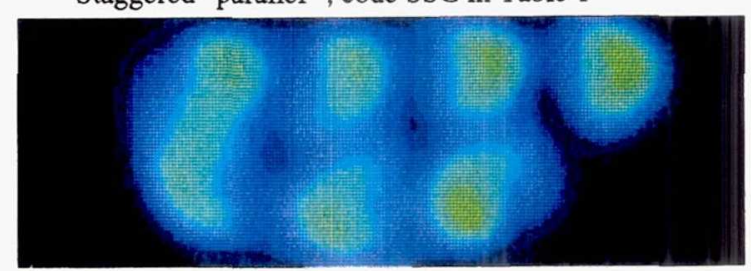

Staggered "crossed" , code SSH in Table 1

Figure 9b: Concentration Distributions of Opposed Slanted Slots at $\mathrm{J}=16, \mathrm{~S} / \mathrm{H}=0.5 . \mathrm{X} / \mathrm{H}=0.83$ 


\section{Time-Resolved Measurements}

$U_{S}$ is a useful performance measure which allows rough screening of various injector configurations. To further assess performance, time-resolved data can be used in conjunction with a chemical model to estimate weighted, mean pollutant formation rates. Absent a chemical calculation, the statistical properties of the flowfield might be useful for differentiating those configurations with low, but comparable, values of $U_{S}$.

Time-resolved measurements were made for opposed inline (code SSE in Table 1) and opposed staggered (code SSG in Table 1) slanted slots at $J=36$ and $X / H=0.33$. For both configurations the data set is comprised of 300 images. Mean concentration distribution, a typical instantaneous concentration distribution, the rms concentration fluctuation distribution, and the relative concentration fluctuation distribution are shown in Fig 10 . Based on the average concentration distribution only, the staggered configuration was shown to have the lower value of $U_{S}$. A comparison of the rms concentration distribution leads to the same conclusion. The fluctuation intensity distribution shows higher fluctuations for the inline configuration, particularly between injectors, indicating that the spacing is too large, as predicted by Eq. 2 .

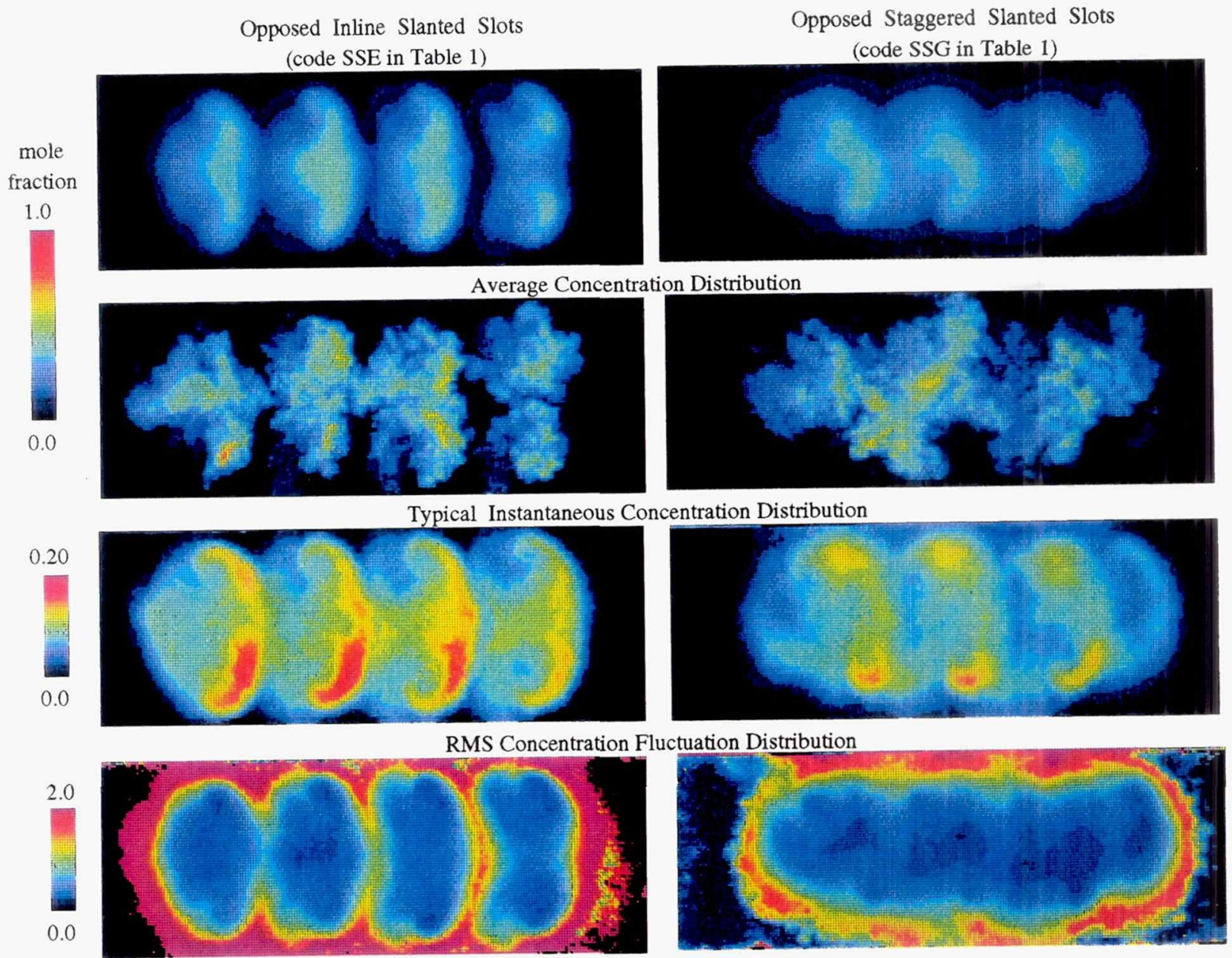

Relative Concentration Fluctuation Distribution

Figure 10: Concentration Distributions obtained from Time-resolved Measurements of the Flow through Opposed Inline $45^{\circ}$ Slanted Slots, $\mathrm{J}=36, \mathrm{X} / \mathrm{H}=0.33$ 
Pdf's of time-resolved and local ensemble average, "spatial", distributions for opposed in-line jets (code SSE in Table 1) are compared in Fig. 11. Areas under the curves are unity. The instantaneous (Fig. 11a-c) and spatial (Fig. 11d) pdf's are similar. As would be expected, the range of fluctuations is greater in the time-resolved data; ensemble averaging truncates the distribution, consistent with a lower value of unmixedness, and the maximum measured mole fraction is reduced from about 0.60 to 0.45 . The results indicate that much of the mixture inhomogeneity can be attributed to mean gradients and, the screening of configurations on the basis of ensemble averaged data should be justified in most cases.
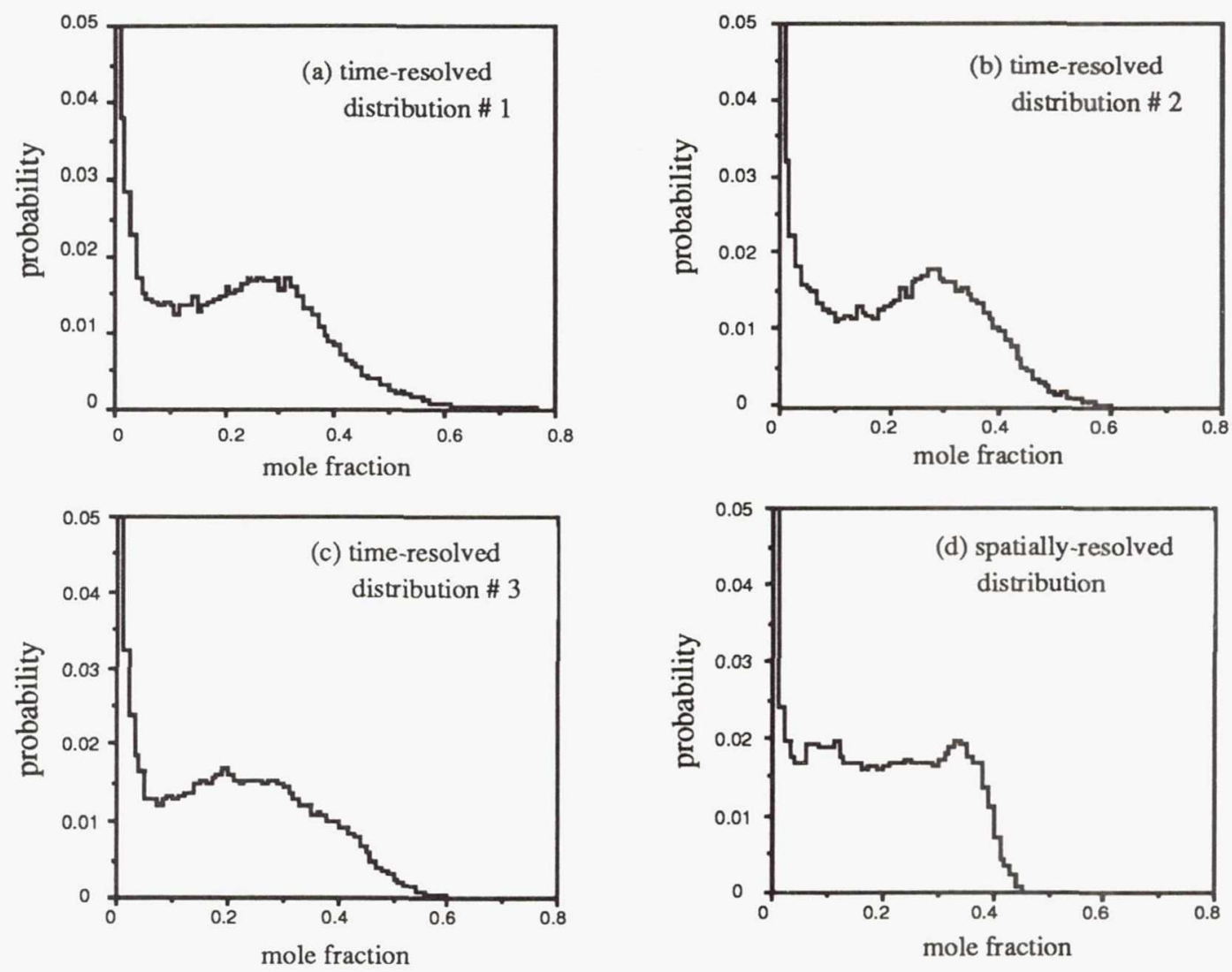

Figure 11: Probability Density Functions of Time-resolved (11a-c) and Spatially Averaged (11d) Concentration Distributions of Opposed Inline Slanted Slots , $\mathrm{J}=36$ and $\mathrm{X} / \mathrm{H}=0.33$ (code SSE in Table 1) 
Spatial pdf's for inline slanted slots (code SSE in Table 1) and round holes (code RHD in Table 1) at $J=36$ are presented in Fig's. 12 and 13, respectively. The mole fraction of the fully mixed fluid, $\mathrm{C}_{\mathrm{avg}}$, is also indicated. It is seen that, (1) the range of the fluctuations is greater with slot injection and, (2) the probability density representing pure mainstream fluid (i.e. mole fraction $=0$ ) is more persistent in the slot configuration. In the round hole configuration, the peak at mole fraction $=0$ is starting to decrease at $\mathrm{X} / \mathrm{H}=1.06$. The pdfs are consistent with Fig. 6 which indicates that $U_{\mathrm{S}}$ is similar for both configurations.

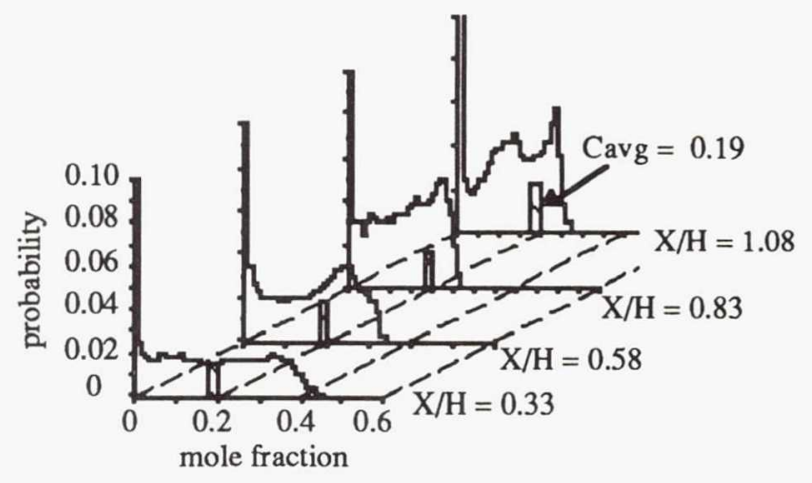

Figure 12: Probability Density Function for Opposed Inline Slanted Slots at $\mathrm{J}=36$ (code SSE in Table 1)

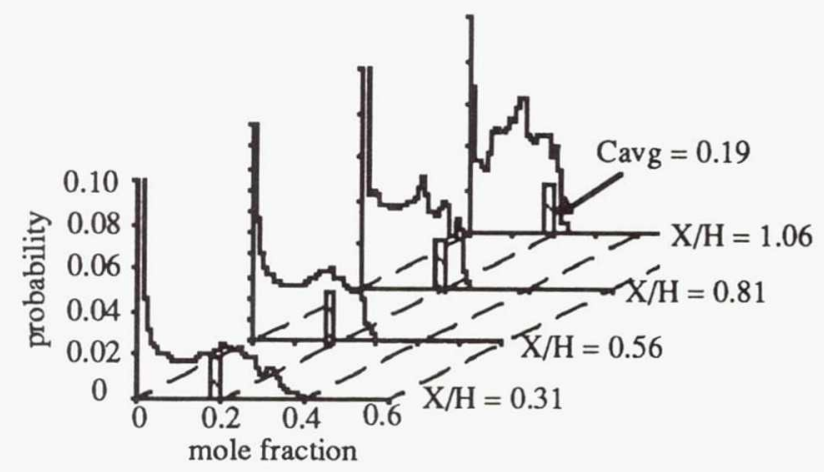

Figure 13: Probability Density Function for Opposed Inline Round Holes at $\mathrm{J}=36$ (code RHD in Table 1)
Pdf's of the opposed staggered slanted slot configuration ( code SSG in Table 1) are shown in Fig. 14. $\mathrm{U}_{\mathrm{S}}$ is considerably lower for this configuration (Fig 8), but despite lower unmixedness, the range of mole fractions is similar to the inline configuration. The initial mixing rate is more rapid than in the opposed inline slot configuration (code SSE in Table 1), as reflected in the narrower distribution away from the peak representing mainstream fluid at mole fraction $=0$. The staggered slot pdf has a bi-modal distribution with little qualitative change occurring with downstream position. Thus, most of the mixing occurs within the first inch of travel; the pdf's at $\mathrm{X} / \mathrm{H}=0.83$ and 1.08 are nearly identical.

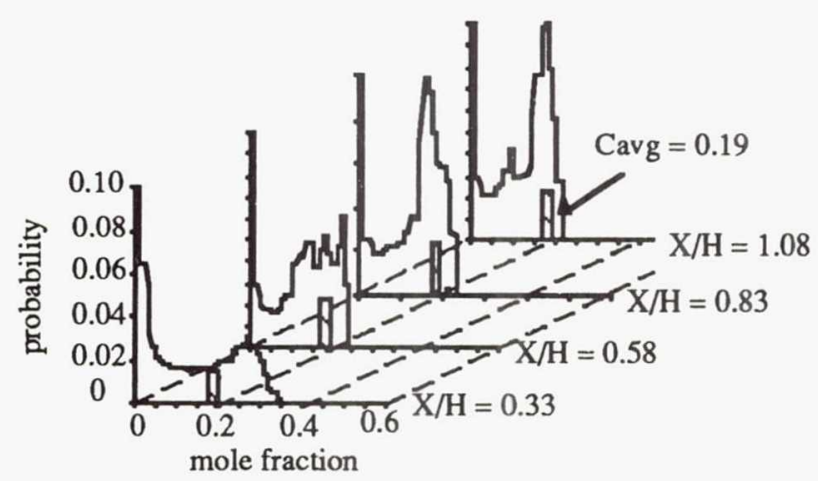

Figure 14: Probability Density Function for Opposed Staggered Slanted Slots at $\mathrm{J}=36$ (code SSG in Table 1) 


\section{Spatial Covariance}

Information about turbulence scales is essential in modeling, as mentioned previously. Turbulence length scales can be estimated from the covariance of the concentration fluctuations. Obtaining this parameter is possible since timeresolved, simultaneous multi-point measurements were acquired. Covariance was calculated relative to a point located at the middle of the duct for opposed inline slanted slots (code SSE in Table 1) at $\mathrm{X} / \mathrm{H}=0.33$ and $\mathrm{J}=36$. The fluctuations were found to be correlated around the selected point to a distance of about 0.6 inches, indicating that the average size of the vortical structures is about $1 / 6$ of the duct height. The spacing between the peaks of adjacent correlated fluctuations corresponds to the characteristic length scale of the flow. Away from the selected point only a weak correlation was found at a distance of 0.20 inches, which is the estimate of the turbulent length scale.

\section{$\underline{\text { Conclusions }}$}

- Momentum-flux ratio and orifice geometry affect mixing performance significantly.

- Mixing rate increases continuously with momentum-flux ratio.

- The coupling between momentum-flux ratio and orifice spacing at the optimum mixing rate, given by Eq. 2 , applies to slanted slots, however blockage is also an important parameter for non-symmetrical orifices.

- Mixing rate is a function of slot orientation.

- At $\mathrm{S} / \mathrm{H} \geq 0.5$ the mixing rate for slanted slots and round holes is equivalent, although jet structure is different.

- Time-resolved measurements support conclusions based on $U_{S}$.

- The PDF's indicate little change in planar mixture distribution with distance.

\section{Acknowledgments}

This work was supported by NASA Contract NAS325952, Task Order \#2.

\section{References}

1. Hatch, M.S., Sowa, W.A., Samuelsen, G.S., Holdeman, J.D., "Jet Mixing Into a Heated Cross Flow in a Cylindrical Duct: Influence of Geometry and Flow Variations," AIAA Paper 92 0773, Reno, Nevada, Jan. 6-9, 1992 (also NASA TM 105390).

2. Holdeman, J.D., "Mixing of Multiple Jets with a Confined Subsonic Crossflow; Summary of NASA Supported Experiments and Modeling," AIAA Paper 91-2458, Sacramento, CA., June 1991 (also NASA TM 104412).

3. Long, M.L., Chu, B.T., and Chang, R.K., "Instantaneous Two-Dimensional Gas Concentration Measurements by Light Scattering," AIAA Journal, Vol. 19, Sept 1981, pp. 1151-1157.

4. Shaw, R.J., "Engine Technology Challenges for a $21 \mathrm{st}$ Century High Speed Civil Transport," AIAA 10th International Symposium on Air Breathing Engines, September 1-6, 1991 (also NASA TM 104361).

5. Smith, C.E., and Talpallikar, M.V., Holdeman, J.D., "A CFD Study of Jet Mixing in Reduced Areas for Lower Combustor Emissions," AIAA Paper 91-2460, Sacramento, CA., June 1991 (also NASA TM 104411).

6. Talpallikar, M.V., Smith, C.E., Lai, M.C., and Holdeman, J.D., "CFD Analysis of Jet Mixing in Low Nox Flametube Combustors," ASME Paper 91-GT-217, Orlando, Fl, June 1991 (also NASA TM 104466).

7. Vranos, A. and Liscinsky, D.S., "Planar Imaging of Jet Mixing in Crossflow," AIAA Journal, 26, 11, November 1988, pp 1297-98.

8. Vranos, A., Liscinsky, D.S., True, B., and Holdeman, J.D., "Experimental Study of Cross-Stream Mixing in a Cylindrical Duct," AIAA Paper 91-2459, Sacramento, CA., June 24-27, 1991 (also NASA TM 105180). 
Public reporting burden for this collection of information is estimated to average 1 hour per response, including the time for reviewing instructions, searching existing data sources, gathering and maintaining the data needed, and completing and reviewing the collection of information. Send comments regarding this burden estimate or any other aspect of this collection of information, including suggestions for reducing this burden, to Washington Headquarters Services, Directorate for information Operations and Reports 1215 Jefferson Davis Highway, Suite 1204, Arlington, VA 22202-4302, and to the Office of Management and Budget, Paperwork Reduction Project (0704-0188), Washington, DC 20503.

\begin{tabular}{|l|c|c|}
\hline 1. AGENCY USE ONLY (Leave blank) & $\begin{array}{c}\text { 2. REPORT DATE } \\
1992\end{array}$ & $\begin{array}{r}\text { 3. REPORT TYPE AND DATES COVERED } \\
\text { Technical Memorandum }\end{array}$ \\
\hline
\end{tabular}

\section{TITLE AND SUBTITLE}

5. FUNDING NUMBERS

Experimental Study of Cross-Stream Mixing in a Rectangular Duct

D.S. Liscinsky, B. True, A. Vranos, and J.D. Holdeman

WU-537-02-20

\section{PERFORMING ORGANIZATION NAME(S) AND ADDRESS(ES)}

National Aeronautics and Space Administration

Lewis Research Center

Cleveland, Ohio 44135-3191

\section{SPONSORING/MONITORING AGENCY NAMES(S) AND ADDRESS(ES)}

National Aeronautics and Space Administration

Washington, D.C. 20546-0001
8. PERFORMING ORGANIZATION REPORT NUMBER

E-7082

10. SPONSORING/MONITORING AGENCY REPORT NUMBER

NASA TM-105694

AIAA-92-3090

\section{SUPPLEMENTARY NOTES}

Prepared for the 28th Joint Propulsion Conference and Exhibit cosponsored by AIAA, SAE, ASME, and ASEE, Nashville, Tennessec, July 6-8, 1992. D.S. Liscinsky and B. True, United Technologies Research Center, East Hartford, Connecticut, 06108; A. Vranos, A.B. Research Associates, South Windsor, Connecticut, 06074; J.D. Holdeman, NASA Lewis Research Center. Responsible person, J.D. Holdeman, (216) 433-5846.

12a. DISTRIBUTION/AVAILABILITY STATEMENT

12b. DISTRIBUTION CODE

Unclassified - Unlimited

Subject Category 07

\section{ABSTRACT (Maximum 200 words)}

An experimental investigation of non-reacting cross-stream jet injection and mixing in a rectangular duct has been conducted with application to a low emissions combustor. Planar digital imaging was used to measure concentration distributions in planes perpendicular to the duct axis. Mixing rate was measured for $45^{\circ}$ slanted slot and round orifice injectors. It is shown that (1) mixing improves continuously with increasing momentum-flux ratio, (2) given a momentum-flux ratio, there is an optimum, orifice spacing, (3) mixing is more dependent on injector geometry than mass flow ratio, (4) mixing is influenced by relative slot orientation, and (5) jet structure is different for round holes and slanted slots injectors. The utility of acquiring multipoint fluctuating properties of the flow field is also demonstrated.

\begin{tabular}{|l|l|l|}
\hline $\begin{array}{l}\text { 14. SUBJECT TERMS } \\
\text { Dilution; Jet mixing flow; Gas turbine; Combustion chamber; Emissions }\end{array}$ \\
$\begin{array}{c}\text { 17. SECURITY CLASSIFICATION } \\
\text { OF REPORT } \\
\text { Unclassified }\end{array}$ & $\begin{array}{c}\text { 18. SECURITY CLASSIFICATION } \\
\text { OF THIS PAGE } \\
\text { Unclassified }\end{array}$ & $\begin{array}{c}\text { 19. SECURITY CLASSIFICATION } \\
\text { OF ABSTRACT } \\
\text { Unclassified }\end{array}$ \\
\hline
\end{tabular}

15. NUMBER OF PAGES

12

16. PRICE CODE

$\mathrm{AO3}$

20. LIMITATION OF ABSTRACT

